\title{
Long-term outcome of decompressive surgery for Lumbar spinal stenosis in octogenarians
}

\author{
Shay Shabat $\cdot$ Zeev Arinzon $\cdot$ Yoram Folman $\cdot$ Josef Leitner · \\ Rami David · Evgeny Pevzner · Reuven Gepstein · Pekarsky Ilya • \\ Ishay Shuval
}

Received: 3 August 2006/Revised: 4 August 2007/Accepted: 16 September 2007/Published online: 17 October 2007

(c) Springer-Verlag 2007

\begin{abstract}
The purpose of our prospective study is to evaluate the surgical outcome among patients aged 80 years and above, who underwent surgery for lumbar spinal stenosis. We assessed patients' clinical and demographic data, procedures, perioperative complications, preoperative and postoperative pain intensity, basic activities of daily living (BADL), patients' satisfaction, the need for repeated surgery, and overall mortality. Thirty-nine patients more than 80 years of age were operated in our institution in the last decade. Twenty-five of them were followed-up with a mean 36.8 months after the operation. The Barthel index was used to evaluate pre and postsurgery ADL, and the visual analogue scale (VAS) was used to evaluate pain. The satisfaction rate of the patients before and after the operation and the complication rate were also evaluated. A significant reduction in VAS $(P<0.001)$ and a significant increase in the Barthel index $(P<0.001)$ were recorded. Seventy-six percent of the patients were very
\end{abstract}

S. Shabat · Z. Arinzon $(\bowtie) \cdot$ J. Leitner $\cdot$ R. David .

E. Pevzner · R. Gepstein · P. Ilya

Spinal Care Unit, Sapir Medical Center,

48 Tchernichovsky Street, Kfar-Saba 44281, Israel

e-mail: arinzon@walla.co.il

S. Shabat · Z. Arinzon · R. Gepstein

Sackler Medical School, Tel Aviv University,

Ramat-Aviv 69978, Israel

Z. Arinzon - I. Shuval

Meuhedet Health Care System, Kfar Saba 42412, Israel

Y. Folman

Department of Orthopaedics B,

Hillel Yaffe Medical Center, Hadera 38100, Israel

Y. Folman

Technion Medical School, 32000 Haifa, Israel satisfied or somewhat satisfied with the operative results. Fifty-two percent of the patients had complications $(0.9$ complications per patients), however, about half of them were minor. No operative or perioperative mortality was noticed and the overall hospital stay for these elderly patients was 3.6 days on average. Surgery in very old elderly patients is safe and effective in the treatment of spinal stenosis, who did not respond well to the conservative treatment. The surgery did not increase the associated morbidity and mortality and most of the patients benefited from the surgery in terms of reduction in pain, increase in ADL and walking ability and overall increase in the satisfaction rate.

Keywords Elderly · Octogenarians - Spinal stenosis . Satisfaction · Surgery

\section{Introduction}

It is well known that the general population is aging and people are living longer. Rapid demographic changes in the elderly population raise other important concerns such as the healthcare. The proportion of the US population aged 65 and above is predicted to rise from $12 \%$ in 2000 to $20 \%$ in 2030 [1]. The elderly currently account for more than onethird of all healthcare spending, and changes in the longevity and number of elderly persons in the United States are expected to further increase the expenditures [7, 26].

More than 17 million people aged 65 years or above in the United States experience at least one episode of low back pain (LBP) or low extremity pain (LEP) in a calendar year [29]. Six million of these individuals suffer from compromised quality of life because of frequent episodes [29]. 
As more people are living into their eighth decade, medical disorders that used to be associated with a much younger population are now occurring more frequently in these older patients. Aging is not a disease; however, there is a relation between age and disease. Age-related changes in the lumbar spine are physiological processes resulting in degenerative changes that may lead to lumbar spine stenosis [9]. Now lumbar spinal stenosis is one of the more frequent condition seen in any orthopedic or neurosurgical practice [6, 30]. Acquired degenerative spinal stenosis usually develops in the seventh decade. Symptoms usually consist of back, buttock, and/or leg pain, with lower-limb sensory and motor deficits. The low back pain (LBP) and low extremity pain (LEP) most consistent with lumbar stenosis usually worsens with ambulation. Patients with lumbar spinal stenosis usually report difficulty in walking long distances, as they were able to do before the stenosis. Spinal stenosis has been recognized as a disorder that is complex, challenging to treat, and associated with wideranging adverse consequences, including physical disability [16], psychosocial disruption which manifests as depression, anxiety, insomnia and social isolation [4, 8, 13, 21], increased use of healthcare resources [3], and may lead to exacerbation of comorbid diseases.

Nonsurgical treatment, such as physiotherapy, analgesic drugs or NSAID and epidural steroids injection is effective in some of the cases. Failure of conservative treatment is an indicator to consider surgical intervention. Surgical treatment is usually performed in patients with moderateto-severe limitation and/or patients with progressive limitation of basic activities of daily living, and only after comparison between patients' complaints and clinical and radiological examinations.

A review of the literature demonstrates conflicting results regarding the outcome of lumbar spine decompression surgery for spinal stenosis in the elderly $[2,10,14$, 18, 20, 24, 27, 30].

The purpose of our study is to evaluate the surgical outcome among patients aged 80 years and above who underwent surgery for lumbar spinal stenosis.

\section{Materials and methods}

\section{Subjects}

During the period from January 1991 to December 2000, 39 patients aged 80 years and above underwent decompressive surgery for lumbar spinal stenosis. The mean age on the time of surgery was 83.95 years (range 8091 years). Twenty-three patients $(59 \%)$ were female and 16 patients $(41 \%)$ were males. A total of $25(64 \%)$ patients were contacted at the time of patient follow-up: six patients died and eight patients were lost to follow-up. The death of the six patients was not related to the operation itself. Patients died from unrelated causes 1-5 years after the operative treatment. No death occurred in the first year. The patients who were lost to follow-up were alive, but did not accept our invitation to arrive for follow-up since they were living relatively far away from our institution. The mean time that elapsed from surgery until patient follow-up was 36.8 months (range 12-72) and mean age on follow-up was 86.6 years (range $82-90$ years). The clinical and demographic characteristics of the study population are shown in Table 1 . Three patients $(8 \%)$ had undergone previous spine surgery: two patients underwent decompressive laminectomy and one patient underwent discectomy.

The diagnosis of lumbar spinal stenosis was made on the basis of clinical and radiological evidences. The mean durations of conservative treatment before surgery were 4.05 months (range 2-7 months). Indications for surgery included progressive limitation in basic activities of daily living, severe pain, or both. Only patients with stenosis due to degenerative spondylosis were included. Patients with degenerative spondylolysthesis or degenerative scoliosis or instability (which was evaluated by flexion-extension $\mathrm{X}$ rays) were excluded from the study. Patients who had fusion in their lumbar spine were excluded from this study.

All patients underwent decompression of the spinal canal and foramina. A central decompression with complete laminectomy was done but the facet joints were not removed. In cases of facet removal and instability formation, the patients underwent fusion and therefore were excluded from the study. In all the cases the foramina were decompressed using Kerrisson rongour. In cases where the stenosis was due to a large herniated disc, a discectomy was performed.

\section{Assessment of patients}

All patients were interviewed on admission using a structured questionnaire. A database was compiled using inpatient and outpatient medical records. Data collection included age, gender, associated comorbidity status, clinical presentation of spinal stenosis, type and duration of conservative treatment, type of the surgery, surgical levels, number of decompressed levels, duration of anesthesia, and postanesthesia care units (PACU) recovery, adverse events, and length of stay in hospital. An adverse event was defined as any event related to surgery or exacerbation in chronic disease during hospitalization and period up to 1 month after discharge from hospital.

The intensity of the pain was described using the visual analogy score (VAS) and compared before surgery and 
Table 1 General characteristics of patients before surgery

\begin{tabular}{|c|c|}
\hline & Mean $\pm \mathrm{SD}(n / \%)$ \\
\hline \multicolumn{2}{|l|}{ Gender } \\
\hline Female & $19(76)$ \\
\hline Male & $6(24)$ \\
\hline \multicolumn{2}{|l|}{ Marital status } \\
\hline Married & $8(32)$ \\
\hline Widowed & $11(44)$ \\
\hline Live alone & $6(24)$ \\
\hline BMI & $26.4 \pm 3.8$ \\
\hline Mean number of comorbid disease & $4.0 \pm 1.5$ \\
\hline Cardiovascular diseases & $21(84)$ \\
\hline HTN & $18(72)$ \\
\hline IHD & $13(52)$ \\
\hline $\mathrm{CHF}$ & $5(20)$ \\
\hline $\mathrm{AF}$ & $3(12)$ \\
\hline $\mathrm{s} / \mathrm{p}$ MI & $3(12)$ \\
\hline s/p CABG & $1(4)$ \\
\hline Osteoarthritis & $9(36)$ \\
\hline Peptic disease & $5(20)$ \\
\hline COPD & $4(16)$ \\
\hline $\mathrm{DM}$ & $3(12)$ \\
\hline Hypothyroidism & $3(12)$ \\
\hline PVD & $3(8)$ \\
\hline Asthma & $3(8)$ \\
\hline VAS & $8.8 \pm 1.9$ \\
\hline ADL (Barthel index) & $62.8 \pm 11.5$ \\
\hline ASA & $2.3 \pm 0.5$ \\
\hline 1 & $2(8)$ \\
\hline 2 & $16(64)$ \\
\hline 3 & $7(28)$ \\
\hline \multicolumn{2}{|l|}{ Type of surgery } \\
\hline Laminectomy & $16(64)$ \\
\hline Discectomy & $1(4)$ \\
\hline Both & $8(32)$ \\
\hline Mean decompressed levels & $2.2 \pm 0.8$ \\
\hline Single level & $6(24)$ \\
\hline Two levels & $16(64)$ \\
\hline Three levels & $3(12)$ \\
\hline
\end{tabular}

during follow-up. Activities of daily living were evaluated during follow-up using the Barthel index comparing preoperative with follow-up data. The Barthel index is a simple and yet validated method widely used for assessing activities of daily living. It is composed of ten tasks used for the basic activities of daily living including feeding, bathing, grooming, dressing, control of bowels and bladder, toilet use, transference, mobility, and climbing stairs. The total Barthel score ranged from 0 to 100 points, with 0 being totally dependent in activities of daily living, and 100 being totally independent in these activities. The limitation in walking distance was graded as less than $50 \mathrm{~m}$, less than $500 \mathrm{~m}$ but more than $50 \mathrm{~m}$, less than $2,000 \mathrm{~m}$ but more than $500 \mathrm{~m}$, and more than $2,000 \mathrm{~m}$.

The American Society of Anesthesiologists (ASA) rating scale was used to assess the severity of health problems at the time of admission. This rating scale categorizes patients as follows: category 1, normal and healthy; category 2 , mild systemic disease; category 3 , severe no incapacitating systemic disease; category 4 , severe incapacitating systemic disease with a constant threat to life; and category 5 , moribund.

Adverse events and later occurring complications were identified during hospitalization and up to 90 days after discharge from the hospital.

Preoperative expectations were assessed by a single question: "do you have low, or high, expectations to have a successful surgical treatment?" to which patients answered with "low/high". Upon termination of the study, patients were asked whether they were "very or somewhat satisfied", or "somewhat or very dissatisfied". An independent observer who was not involved in the care of the patients recorded the data concerning the patient's satisfaction.

\section{Statistical analysis}

The nonparametric data among the groups of patients were analyzed using the Kruskal-Wallis test and the Chi square test. The Yates correction for Chi square was done whenever any of the cells in a $2 \times 2$ table was less than five and the unadjusted Chi square was $\chi^{2} 3.84(P<0.05)$. Longterm survival was estimated by the life-table method.

Data were expressed as mean $\pm \mathrm{SD}$, rates, or numbers. The statistics were done using the SPSS 12.0 programme.

\section{Results}

The mean numbers of comorbid disease per patient were 4.04. Eighty-four percent of patients suffered from at least one cardiovascular disease. Cardiovascular disease were subdivided into arterial hypertension (72\%), ischemic heart disease $(52 \%)$, congestive cardiac failure $(20 \%)$, chronic atrial fibrillation (12\%), previous myocardial infarction (12\%), and coronary artery bypass graft $(4 \%)$. Other coexisting diseases included osteoarthritis (36\%), peptic disease (20\%), chronic obstructive lung disease (16\%), diabetes mellitus (12\%), hypothyroidism (12\%), peripheral vascular disease (8\%), and bronchial asthma (8\%).

The mean duration of symptoms before the operation by patients' reports was 52.6 months (range 2-360 months). Before the surgery, the patient physical status was recorded 
as class I, II and III, under the American Society of Anesthesiologists (ASA) classification, in 8, 64 and 28\% of patients, respectively. The mean anesthesia time (time from intubation to extubation) and the mean surgical time (time from skin incision to skin closure) were 79.1 and $58.4 \mathrm{~min}$, respectively. The mean PACU time was $83.5 \mathrm{~min}$.

The mean decomposed levels were 2.2. Twenty-four percent of patients underwent decompression on a singlelevel. Surgical decompressions of two and three levels were performed in 64 and $12 \%$ of patients, respectively. L4-5 was the predominant surgical level (53\%) followed by L3-4 (28\%) and L5-S1 (15\%).

Postoperative complications were reported in $52 \%$ of the patients, with a mean of 0.9 complications per patient (Table 2). During hospitalization, no cases of surgery related mortality and neurological injury were found. CVA was reported in one of the patients, 1 month after discharge. The mean length of stay time was 3.6 days (range 2-9 days). Postoperative complications were unrelated to the existing comorbitities and ASA class. No dural tears were encountered in this series even in the patients who were operated for the second time.

The results of the Kaplan-Meier survivorship survival analysis are shown graphically in Fig. 1. The survival rates for 2, 4, and 6 years from the operation were 94, 80.8 and $72.7 \%$, respectively.

A significant improvement in overall pain perception was recorded following the surgery. The patients reported VAS score of $8.84+1.91$ prior to surgery, which improved to $3.6+2.35$ on latest follow-up $(P<0.001)$.

Furthermore, patients reported a significant $(P<0.001)$ subjective improvement in the ability to perform daily activities (the Barthel index improved from $62.8 \pm 11.46$ points before the surgery to $77.0 \pm 11.9$ points after the

Table 2 Number of post surgical complications

\begin{tabular}{ll}
\hline Complication & $N(\%)$ \\
\hline Urinary retention & $6(24)$ \\
UTI & $5(20)$ \\
Bronchospasm & $3(12)$ \\
Wound infection & $2(8)$ \\
PAF & $2(8)$ \\
Delirium & $2(8)$ \\
Depression & $2(8)$ \\
CVA & $1(4)$ \\
Number of complication per patient & \\
0 & $12(48)$ \\
1 & $6(24)$ \\
2 & $5(20)$ \\
3 & $1(4)$ \\
4 & $1(4)$ \\
\hline
\end{tabular}

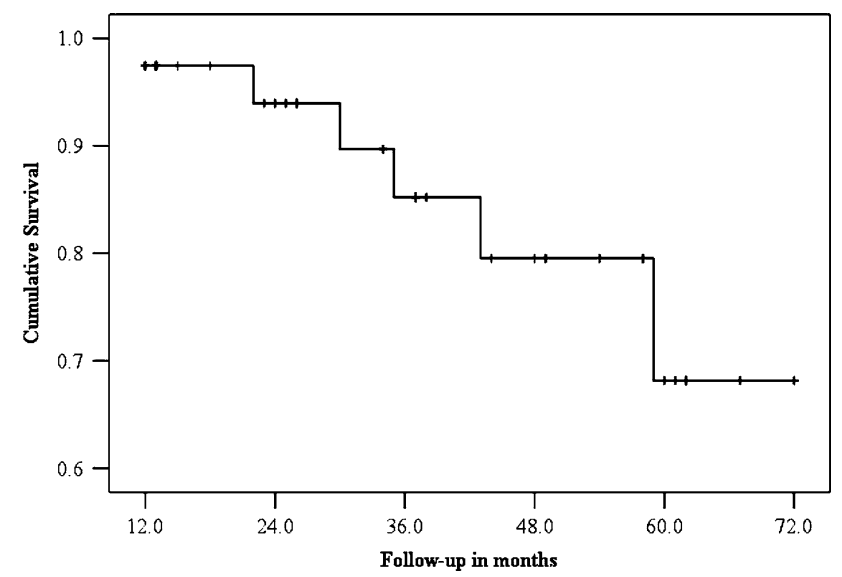

Fig. 1 Kaplan-Meier survival curve

surgery $(P<0.001)$. The number of patients who had limitation in walking distance upto $50 \mathrm{~m}$ decreased by $28 \%$ $\left(\chi^{2}=3.74, P=0.053\right)$, and the number of patients having no walking limitation increased by $32 \%\left(\chi^{2}=5.37\right.$, $P=0.02)$. In addition, a significant decrease in analgesic drug consumption, physiotherapy and alternative medicine treatment was noticed (Figs. 2, 3).

Sixty-eight percent of the patients had a prior belief in the surgical success. On the time of follow-up, $76 \%$ of patients reported that they were very $(4 / 25)$ and somewhat $(15 / 25)$ satisfied with overall surgical results.

During the follow-up period, re-operation was performed in one patient. The first operation of this patient was a discectomy in the L3-4 level. After 31 months this patient was reoperated because of stenosis at the L4-5 level.

\section{Discussion}

This study investigates 25 consecutive cases of decompressive surgery for lumbar spinal stenosis in patients

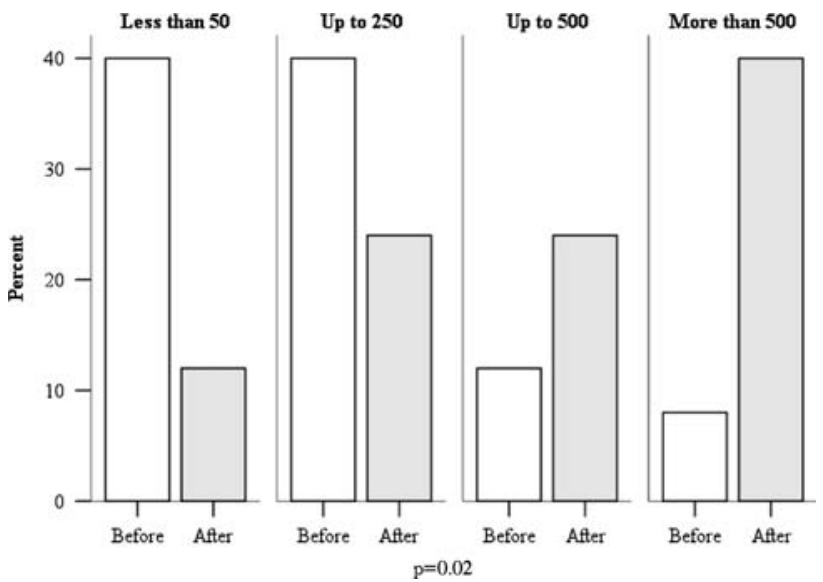

Fig. 2 Walking distance (m) 


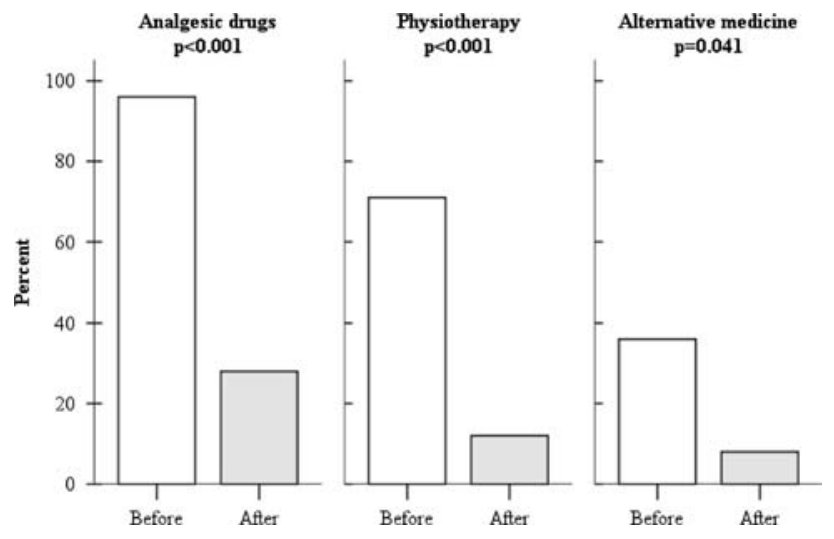

Fig. 3 Treatment before and after operation

80 years of age and older who were contacted and followed-up. The result of this study demonstrates favorable outcome and satisfaction in this patient population. We were able to follow 25 out of 39 patients (64\%). During our 10 -year follow-up period, six of our patients died. However, in contrast with previous reports [5, 25], in the current group, no patient died in the hospital or within the intermediate (less than 3 months) postoperative period.

Furthermore, when assessing the effect of surgery on patient mortality, it is important to compare the mortality rate of the study cohort with that of a matched non-study population. By using life-table survival analysis, we demonstrated that cumulative survival rate in period upto 6 years after the surgery was $72.7 \%$. According to the Israel Central Bureau of Statistics, the mortality rate of patients older than 75 years is approximately $9 \%$ per year. Since our mortality rate was over a 10 -year period it is likely that the surgery did not affect patient mortality.

Although the number of comorbid disease is relatively high (4.0 per patient on average), none of the patients died during the immediate postoperative period. In those with perioperative complications, treatment was successful and none of them required intensive care or prolonged hospital stay. Patients described significant reduction of LBP and LEP and improvement in their ability to perform daily activities.

In our study the mean duration of hospital stay was 3.6 days. This is in contradiction with the report of Vitaz et al. [28] who found an average length of stay of 11.6 days for elderly patients ( $>75$ years) recovering from surgical release of spinal stenosis. This is probably due to a combination of lower comorbidity of our patients and a good operative technique, which yielded almost no major perioperative complications.

Some studies $[15,17]$ found an association between comorbitidy, complication rate and surgical outcome (acceptable outcome in patients with no or only minor comorbidity). Fifty-two percent of our patients suffered from complications. This rate is higher in comparison with other previous studies [5, 10, 22, 25]. However, half of them $(48 \%)$ were minor and did not result in prolonged length of stay. This rate of complications was not correlated to a high ASA class and high number of comorbidities.

Katz et al. [19] reported a satisfaction rate of $75 \%$ for patients who underwent decompression for lumbar spinal stenosis with a mean age 69 years (range 50-92 years). Sanderson and Wood [23] reported excellent or good results in $81 \%$ of their patients ( 65 years of age or older) during an average follow-up of 42 months. In a study similar to ours in terms of ages (mean age 82.2 years, mean follow-up 32.4 months), Galiano et al. [10] with a mean age of patients similar to our series reported a satisfaction rate of $65 \%$ of the operated patients. In our study, with a mean age of 83.7 years at the time of surgery, with a follow-up period of 36.8 months on average (minimal followup of 1 year), $76 \%$ of the operated patients reported that they were very or somewhat satisfied with the surgical results.

Impaired lower extremity functioning and progressive walking difficulties in old age are important predictors of disability and loss of mobility, and are important constituents of frailty, a state characterized by clinical instability and decline in physiologic reserve and significant functional decline $[31,32]$. Our study demonstrates that spinal surgery in elderly patients is an effective medical tool not only in improving quality of life (decrease suffering from pain and in performance of BADL), but also in decreasing the cost of health care (decrease in drug use and other medical service by patients).

In a previous study [11], it was shown that the satisfaction rate did not vary among patients in the different BMI groups, although the percentage of dissatisfaction was higher in patients who were obese. It seems that the functional outcome of lumbar spinal stenosis is influenced by the BMI. We also demonstrated that in the elderly, preoperative expectations reasonably predict their postoperative satisfaction rate [12].

A limitation of the study is the relatively small number of operated patients. In addition, we had a relatively high percentage of dropouts from the study (14 out of the 39 patients $(36 \%)$ were lost to follow-up, six patients because of death and eight patients were lost to follow-up). However, the follow-up period of the 25 patients who were followed was quite long enough. Moreover, in this study no comparison to a young patient group was done.

We conclude that decompressive surgery is a good option for selected patients after 80 years of age who did not respond well to conservative treatment. This treatment did not increase the associated morbidity and mortality and most of the patients benefited from the surgery (up to three 
levels) in terms of reduction in pain, increase in basic activities of daily living and walking ability and overall increase in the satisfaction rate. However, it should be remembered that elderly patients might deteriorate following surgery. Therefore surgery should be done only after measuring the potential benefits of the surgery versus the potential risks.

\section{References}

1. Annual Report of the Board of Trustees of the Federal Old-Age and Survivors Insurance and Disability Insurance Trust Funds (2001) Social Security Administration, Baltimore

2. Caliandro P, Aulisa L, Padua R, Aprile I, Mastantuoni G, Mazza O, Tonali P, Padua L (2005) Quality of life, clinical and neurophysiological picture in patients operated on for lumbar stenosis. Acta Neurochir (Suppl) 92:143-146

3. Carey TS, Evans A, Hadler N, Kalsbeek W, McLaughlin C, Fryer J (1995) Care-seeking among individuals with chronic low back pain. Spine 20:312-327

4. Carr DB, Jacox AK, Chapman CR (1992) Acute pain management: operative or medical procedures and trauma. Clinical practice guideline No. 1. AHCPR Pub. No 92-0032, Agency for Health Care Policy and Research, US Department of Health and Human Services, Rockville

5. Deyo RA, Cherkin DC, Loeser JD, Bigos SJ, Ciol MA (1992) Morbidity and mortality in association with operation on the lumbar spine: the influence age, diagnosis, and procedure. J Bone Joint Surg Am 74:536-543

6. Deyo RA, Mirza SK (2006) Trends and variations in the use of spine surgery. Clin Orthop Relat Res 443:139-146

7. Lubitz J, Beebe J, Baker C (1995) Longevity and medicare expenditures. N Engl J Med 332:999-1003

8. Ferrell BA (1991) Pain management in elderly people. J Am Geriatr Soc 39:64-73

9. Gafrin SR, Herrkowitz HN, Mirkovic S (1999) Instructional course lectures, The American academy of orthopaedic surgeons-spinal stenosis. J Bone Joint Surg Am 81:572-586

10. Galiano K, Obwegeser AA, Gabl MV, Bauer R, Twerdy K (2005) Long-term outcome of laminectomy for spinal stenosis in octogenarians. Spine 30:332-335

11. Gepstein R, Shabat S, Arinzon Z, Berner Y, Catz A, Folman Y (2004) Does obesity affect the results of lumbar decompressive spinal surgery in the elderly? Clin Orthop Relat Res 426:138-144

12. GepsteinR, Arinzon Z, Adunsky A, Folman Y (2006) Decompression surgery for lumbar spinal stenosis in the elderly: preoperative expectations and postoperative satisfaction. Spinal Cord 44(7):427-431

13. Helme RD, Gibson SJ (1997) Pain in the elderly. In: Jensen TS, Turner JA, Wiesenfeld-Hallin Z (eds) Proceedings of the 8th world congress on pain: progress in pain research and management, vol 8. IASP Press, Seattle, pp 919-944

14. Javid MJ, Hadar EJ (1998) Long-term follow-up review of patients who underwent laminectomy for lumbar stenosis: a prospective study. J Neurosurgery 89:1-7

15. Jonsson B, Annertz M, Sjoberg C, Stromqvist B (1997) A prospective and consecutive study of surgical treated lumbar spinal stenosis. Part I Clinical features related to radiographic findings. Spine 22:2932-2937

16. Jonsson B, Stromqvist B (1993) Symptoms and signs of degeneration of the lumbar spine: A prospective, consecutive study of 300 operated patients. J Bone Joint Surg Br 75:381-385

17. Katz JN, Lipson SJ, Brick GW, Grobler LJ, Weinstein JN, Fossel AH, Lew RA, Liang MH (1995) Clinical correlates of patients satisfaction after laminectomy for degenerative lumbar spinal stenosis. Spine 20:1155-1160

18. Katz JN, Lipson SJ, Chang LC, Levine SA, Fossel AH, Liang MH (1996) Seven to 10-year outcome of decompressive surgery for degenerative lumbar spine disorders. Spine 21:92-98

19. Katz JN, Stucki G, Lipson SJ, Fossel AH, Grobler LJ, Weinstein JN (1999) Predictors of surgical outcome in degenerative lumbar spinal stenosis. Spine 24:2229-2233

20. Kilincer C, Steinmetz MP, Sohn MJ, Benzel EC, Bingaman W (2005) Effects of age on the perioperative characteristics and short-term outcome of posterior lumbar fusion surgery. J Neurosurg Spine 3:34-39

21. Magni G, Marchetti M, Moreschi C, Luchini SR (1993) Chronic musculoskeletal pain and depressive symptoms in the national health and nutrition examination: epidemiologic follow-up study. Pain 53:163-168

22. Ragab AA, Fye MA, Bohlman HH (2003) Surgery of the lumbar spine for spinal stenosis in 118 patients 70 years of age or older. Spine 28:348-353

23. Sanderson PL, Wood PL (1993) Surgery for lumbar spinal stenosis in old people. J Bone Joint Surg Br 75:393-397

24. Scholz M, Firsching R, Lanksch WR (1998) Long-term follow up in lumbar spinal stenosis. Spinal Cord 36:200-204

25. Silvers HR, Lewis PJ, Asch HL (1993) Decompressive lumbar laminectomy for spinal stenosis. J Neurosurg 78:695-701

26. Spillman BC, Lubitz J (2000) The effect of longevity on spending for acute and long-term care. N Engl J Med 342:1409-1415

27. Tadokoro K, Miyamoto H, Sumi M, Shimomura T (2005) The prognosis of conservative treatments for lumbar spinal stenosis: analysis of patients over 70 years of age. Spine 30:2458-2463

28. Vitaz TW, Raque GH, Shields CB, Glassman SD (1999) Surgical treatment of lumbar spinal stenosis in patients older than 75 years of age. J Neurosurg 91:181-185

29. Weiner DK, Haggerty CL, Kritchevsky SB, Harris T, Simonsick EM, Nevitt M,Newman A (2003) How does low back pain impact physical function in independent, well-functioning older adults? Evidence from the health $\mathrm{ABC}$ cohort and implications for future. Pain Med 4:311-320

30. Weinstein JN, Bronner KK, Morgan TS, Wennberg JE (2004) Trends and geographic variations in major surgery for degenerative diseases of the hip, knee, and spine. Health Aff (Millwood). Suppl Web Exclusive:VAR81-9

31. Ferrucci L, Bandinelli S, Benvenuti E, Di Iorio A, Macchi C, Harris TB, Guralnik JM (2000) Subsystems contributing to the decline in ability to walk: bridging the gap between epidemiology and geriatric practice in the CHIANTI study. J Am Geriatr Soc 48:1618-1625

32. Guralnik JM, Ferrucci L, Simonnsick EM, Salive ME, Wallace RB (1995) Lower-extremity function in persons over the age of 70 years as a predictor of subsequent disability. N Engl J Med $332: 556-561$ 